\title{
Magnetism and Néel skyrmion dynamics in $\mathrm{GaV}_{4} \mathrm{~S}_{8-y} \mathrm{Se}_{y}$
}

\author{
T. J. Hicken $\odot,{ }^{1}$ S. J. R. Holt $\odot,{ }^{2}$ K. J. A. Franke $\odot,{ }^{1, *}$ Z. Hawkhead $\odot,{ }^{1}$ A. Štefančičc, ${ }^{2, \dagger}$ M. N. Wilson, ${ }^{1}$ M. Gomilšek $\odot,{ }^{1,}$ \\ B. M. Huddart ${ }^{1}{ }^{1}$ S. J. Clark, ${ }^{1}$ M. R. Lees $\odot,{ }^{2}$ F. L. Pratt $\odot,{ }^{3}$ S. J. Blundell $\odot,{ }^{4}$ G. Balakrishnan ${ }^{\circ},{ }^{2}$ and T. Lancaster ${ }^{1}$ \\ ${ }^{1}$ Centre for Materials Physics, Durham University, Durham DH1 3LE, United Kingdom \\ ${ }^{2}$ Department of Physics, University of Warwick, Coventry CV4 7AL, United Kingdom \\ ${ }^{3}$ ISIS Pulsed Neutron and Muon Facility, STFC Rutherford Appleton Laboratory, Harwell Oxford, Didcot OX11 OQX, United Kingdom \\ ${ }^{4}$ Department of Physics, Oxford University, Clarendon Laboratory, Parks Road, Oxford OX1 3PU, United Kingdom
}

(Received 19 March 2020; revised 20 May 2020; accepted 4 June 2020; published 1 July 2020)

\begin{abstract}
We present an investigation of the influence of low levels of chemical substitution on the magnetic ground state and Néel skyrmion lattice (SkL) state in $\mathrm{GaV}_{4} \mathrm{~S}_{8-y} \mathrm{Se}_{y}$, where $y=0,0.1,7.9$, and 8. Muon-spin spectroscopy ( $\mu \mathrm{SR}$ ) measurements on $y=0$ and 0.1 materials reveal the magnetic ground state consists of microscopically coexisting incommensurate cycloidal and ferromagnetic environments, whereas chemical substitution leads to the growth of localized regions of increased spin density. $\mu \mathrm{SR}$ measurements of emergent low-frequency skyrmion dynamics show that the SkL exists under low levels of substitution at both ends of the series. Skyrmionic excitations persist to temperatures below the equilibrium SkL in substituted samples, suggesting the presence of skyrmion precursors over a wide range of temperatures.
\end{abstract}

DOI: 10.1103/PhysRevResearch.2.032001

Chemical substitution is well known for stabilizing exotic states of matter from high- $T_{\mathrm{c}}$ superconductivity in Mott insulators [1] to hidden magnetic order in heavy-fermion compounds [2-4] and nonperturbative strongly correlated Kondo states in itinerant systems [5-7]. The influence of chemical substitution on topological defects, such as magnetic skyrmions [8,9], has been shown to be particularly pronounced, with substitution of just a few percent of the magnetic ions increasing the stability and lifetime of skyrmions [10] and modifying their creation/annihilation energy barriers [11]. The study of the effects of low levels of chemical substitution in bulk skyrmion materials has concentrated on materials hosting Bloch skyrmion lattice (SkL) states [12-16] as experimental realizations of Néel skyrmions in bulk materials are rare [17-20]. We have previously studied the influence of high levels of chemical substitution on the Néel SkL compounds $\mathrm{GaV}_{4} \mathrm{~S}_{8}$ and $\mathrm{GaV}_{4} \mathrm{Se}_{8}$ by investigating $y=2$ and 4 compositions in the $\mathrm{GaV}_{4} \mathrm{~S}_{8-y} \mathrm{Se}_{y}$ series [21,22] and showed

\footnotetext{
* Present address: Department of Materials Science and Engineering, University of California, Berkeley, Berkeley, California 94720, USA; School of Physics and Astronomy, University of Leeds, Leeds LS2 9JT, United Kingdom.

${ }^{\dagger}$ Present address: Electrochemistry Laboratory, Paul Scherrer Institut, CH-5232 Villigen PSI, Switzerland.

${ }^{\ddagger}$ Present address: Jožef Stefan Institute, Jamova cesta 39, SI-1000 Ljubljana, Slovenia.

Published by the American Physical Society under the terms of the Creative Commons Attribution 4.0 International license. Further distribution of this work must maintain attribution to the author(s) and the published article's title, journal citation, and DOI.
}

that it induces a spin-glass ground state and destroys the Néel SkL state.

Here, we investigate the low-level limit of chemical substitution in the series through muon spin spectroscopy $(\mu \mathrm{SR})$ [23], AC susceptibility [24] and first-principles calculations carried out using density functional theory (DFT) [25-29]. The influence of low levels of substitutions on both the magnetic ground state and the $\mathrm{SkL}$ in $\mathrm{GaV}_{4} \mathrm{~S}_{8-y} \mathrm{Se}_{y}$ is studied by comparing compositions very close to each end of the series, where $y=0.1$ and $y=7.9$, with Néel SkL-hosting $y=0\left(\mathrm{GaV}_{4} \mathrm{~S}_{8}\right)$ and $y=8\left(\mathrm{GaV}_{4} \mathrm{Se}_{8}\right)$ compounds. We find that the ground state of $\mathrm{GaV}_{4} \mathrm{~S}_{8}$ is most sensitive to substitution with significant changes of spin density near substituents. The dynamic signature of skyrmions persists in the substituted materials at both ends of the series with an extended region of emergent low-frequency dynamics evident at low temperatures.

Polycrystalline samples of $\mathrm{GaV}_{4} \mathrm{~S}_{8-y} \mathrm{Se}_{y}$ were synthesized and characterized as described in Refs. [21,22]. Whereas polycrystalline samples are likely to exhibit a different magnetic phase diagram to those of single crystals (whose behavior varies depending on the alignment between applied field and crystallographic axes), we have previously shown that the SkL can be identified unambiguously [21]. AC magnetic susceptibility (Fig. 1) indicates relatively small changes in the position of the phase boundaries in the substituted materials when compared to their pristine counterparts, suggesting the SkL state is still formed. In the substituted systems, the maximum susceptibility is approximately an order of magnitude greater than in the pristine systems, suggesting enhanced dynamics at low frequencies.

To explore the magnetic ground state of the systems, zerofield (ZF) $\mu \mathrm{SR}$ measurements were performed. In $\mathrm{GaV}_{4} \mathrm{~S}_{8}$, 

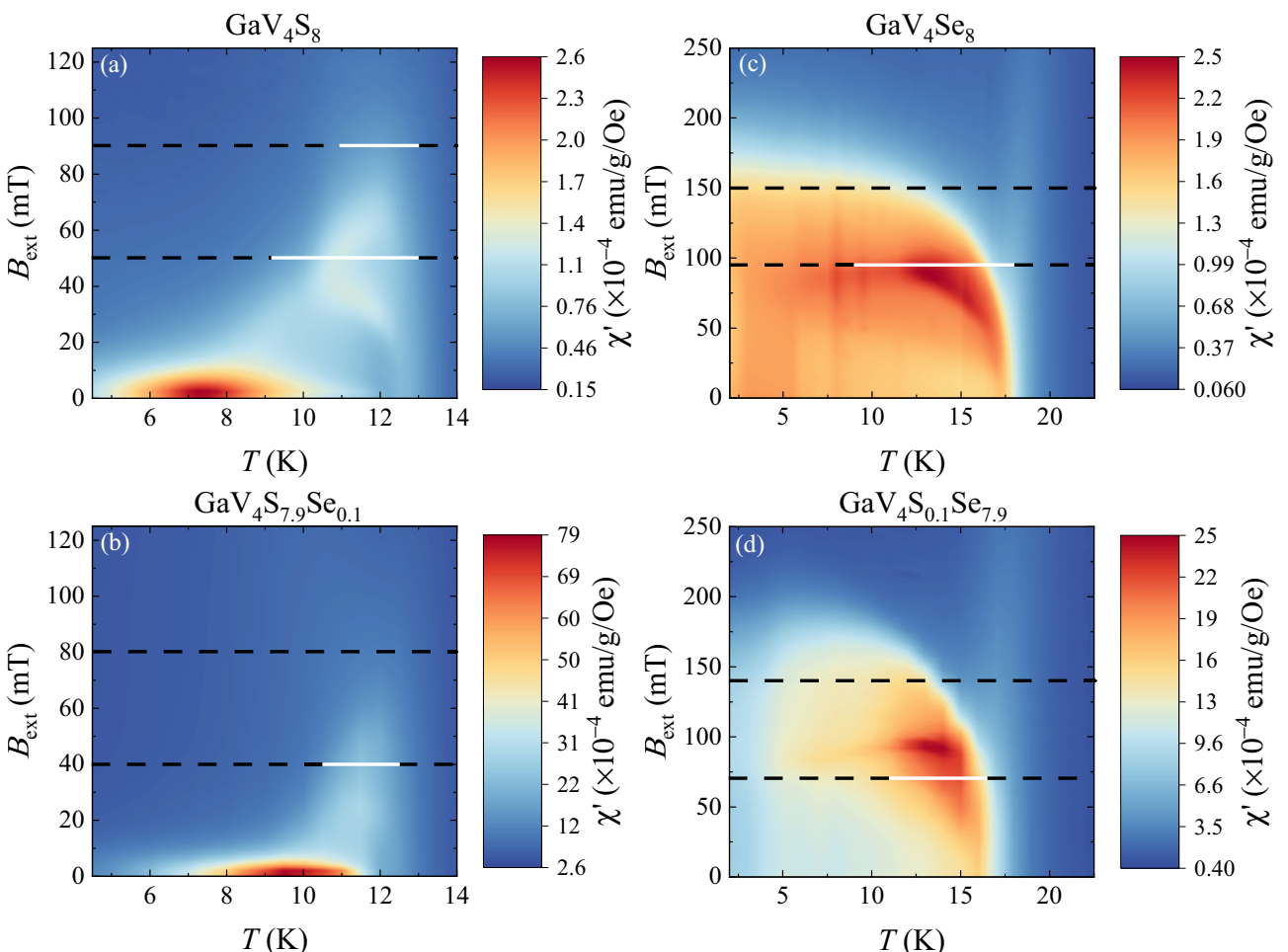

FIG. 1. Real component of AC susceptibility in constant field $B_{\text {ext }}$ for (a) $y=0$ (b) $y=0.1$, (c) $y=8$, and (d) $y=7.9$. Lines indicate fields where $\mu$ SR measurements were performed with white highlighting proposed SkL regions as based on $\mu$ SR measurements (see the main text).

above $T_{\mathrm{c}}=12.7(3) \mathrm{K}$ we find that the muon-spin polarization $P_{z}(t)$ is parametrized [30] by $P_{z}(t)=a e^{-\lambda t}+a_{\mathrm{b}} e^{-\lambda_{\mathrm{b}} t}$, typical of a paramagnet. The first term with amplitude $a$ reflects relaxation at rate $\lambda$ from muons that stop within the sample in the paramagnetic state, whereas the $a_{\mathrm{b}}$ component captures the contributions from muons that stop outside the sample. Fourier transforms (FTs) of $P_{z}(t)$ in the ordered phase $\left(T<T_{\mathrm{c}}\right)$ are shown in Figs. 2(a)-2(c). Simulations of the magnetic field [31,32] at the muon-stopping sites [21] for the ground-state magnetic structures proposed for $\mathrm{GaV}_{4} \mathrm{~S}_{8}$ $[17,22,33]$ show that the distribution most closely resembles the ferromagneticlike (FM*) state [Fig. 3(a)] at low temperatures, and the incommensurate cycloidal (C) state at higher temperatures. At all temperatures, the spectra have features similar to those of both magnetic structures, however, the data cannot be described by a simple sum of the two simulations as would be expected for spatially separated domains of FM* and $\mathrm{C}$ orders. Our data suggest a continuous evolution of the magnetic ground state from $\mathrm{FM}^{*}$ to $\mathrm{C}$ (rather than an abrupt phase transition) where the spins slowly transform from one structure to the other. In fact, this crossover has been suggested to occur via nucleation and growth of solitons [34,35] with the associated cycloidal anharmonicity likely to help explain some of the discrepancies between simulation and experiment. The precise mechanism is likely to depend sensitively on the crystalline anisotropy in the system [36]. Our data, therefore, suggest FM* domains are prevalent at low $T$ with the possibility of solitonlike cycloidal domain walls growing continuously with increasing $T$ until a C-majority phase is realized.
To compare the pristine and substituted systems at temperatures $T<T_{\mathrm{c}}$, the polarization is fitted to

$$
P_{z}(t)=\sum_{i=1}^{n} a_{i} e^{-\Lambda_{i} t} \cos \left(\gamma_{\mu} B_{i} t+\phi_{i}\right)+a_{\mathrm{b}} e^{-\lambda_{\mathrm{b}} t},
$$

where each component with amplitude $a_{i}$, and relaxation rate $\Lambda_{i}$, reflects muons that stop in local field $B_{i}$, and precess with phase offset $\phi_{i}$. For $\mathrm{GaV}_{4} \mathrm{~S}_{8}$, we require only $n=2$, indicating two magnetically distinct components with local field magnitude $B_{i}$. Extracted parameters [Figs. 2(d) and 2(e)] show that $B_{1}$ corresponds with the low-field peak seen in the simulations of both the $\mathrm{FM}^{*}$ and $\mathrm{C}$ states [indicated in Figs. 2(a)-2(c) with a black line] with $B_{2}$ corresponding to the high-field peak (red line). The unusual decrease in $B_{2}$ with decreasing temperature, along with a change in the fraction of muons subject to this magnetic field, reflects the continuous evolution of the magnetic state, providing further evidence for a smooth crossover between the $\mathrm{FM}^{*}$ and the $\mathrm{C}$ states, rather than a sharp phase transition [21]. The crossover region $5 \lesssim T \lesssim 9 \mathrm{~K}$ [shaded in Fig. 2(e)] reflects the most rapid change of spin structure which leads to the enhanced AC susceptibility response seen in Fig. 1(a).

For the $\mathrm{GaV}_{4} \mathrm{~S}_{7.9} \mathrm{Se}_{0.1}$ material, up to $T_{\mathrm{c}}=11.6(2) \mathrm{K} \mathrm{ZF}$ $\mu \mathrm{SR}$ measurements are well parametrized by Eq. (1) with $n=3$, indicating a third magnetically distinct muon environment not observed in $\mathrm{GaV}_{4} \mathrm{~S}_{8}$ [Fig. 2(f)]. Below $T_{\mathrm{c}}$, the amplitudes $a_{i}$ are found to be temperature independent, indicating that $13(5) \%$ of the muons stopping in the sample stop in sites with $B_{1}, 32(3) \%$ in $B_{2}$, and $55(4) \%$ in $B_{3}$. The 

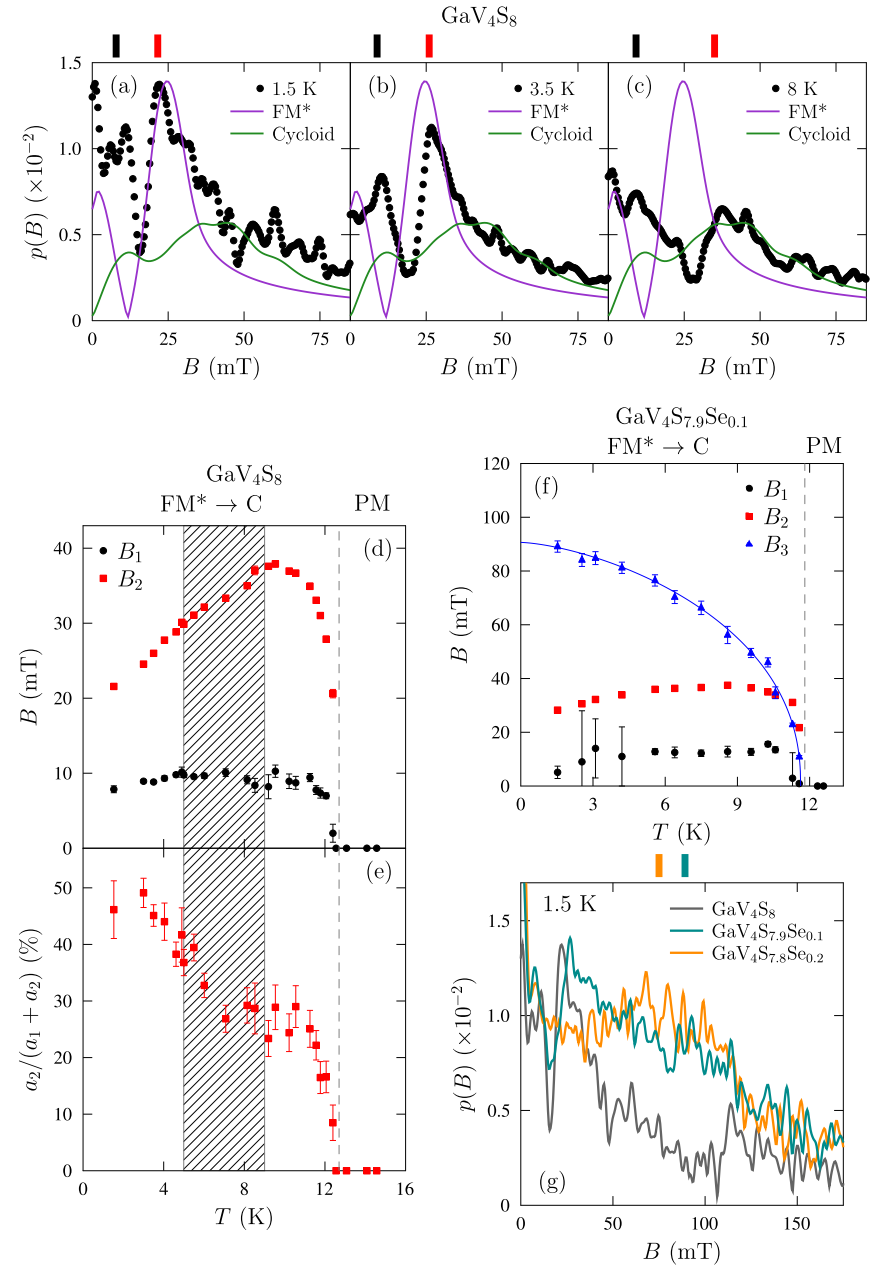

FIG. 2. (a)-(c) Internal magnetic-field distributions $p(B)$ for $\mathrm{GaV}_{4} \mathrm{~S}_{8}$, obtained via the FTs of ZF $\mu$ SR data at several temperatures, compared to simulations of the FM* [Fig. 3(a)] and cycloidal (C) states. Parameters from ZF $\mu \mathrm{SR}$ measurements of $\mathrm{GaV}_{4} \mathrm{~S}_{8-y} \mathrm{Se}_{y}$ for (d) and (e) $y=0$ and (f) $y=0.1$. The extracted internal fields seen in (d) are marked in (a)-(c). (g) $p(B)$ for $y=0, y=0.1$, and $y=0.2$ with the $B_{3}$ component marked for $y=0.1$ and $y=0.2$.

ratio $n_{1} / n_{2}$ in $\mathrm{GaV}_{4} \mathrm{~S}_{7.9} \mathrm{Se}_{0.1}$ is consistent with the $T>8 \mathrm{~K}$ region in $\mathrm{GaV}_{4} \mathrm{~S}_{8}$ where $\mathrm{C}$ order dominates. There are three mechanisms which could explain the appearance of the $B_{3}$ component in $\mathrm{GaV}_{4} \mathrm{~S}_{7.9} \mathrm{Se}_{0.1}$. (i) A change in spin structure. This can be ruled out as $B_{1}$ and $B_{2}$ are very similar in magnitude and $T$ evolution to $\mathrm{GaV}_{4} \mathrm{~S}_{8}$, suggesting similar underlying behavior. (ii) An increase in the magnetic moment $m$. The field at the muon site $B_{i} \propto m$. As $B_{3} / B_{2} \simeq 3$, this would imply an increase in the moment by the same factor, which can again be ruled out as there is no evidence for this in $B_{1}$ and $B_{2}$, or in DC magnetization measurements [32]. (iii) A change in distance $r$ between the spin density and the muon. As $B_{i} \propto 1 / r^{3}$, even a modest change in spin density could lead to dramatic changes in $B_{i}$. We, therefore, suggest that the most likely explanation of the appearance of the $B_{3}$ component is an increase in spin density near the muon sites such that these regions of high magnetic field condense around the substituent. This is supported by the FT of $P_{z}(t)$ for $\mathrm{GaV}_{4} \mathrm{~S}_{7.9} \mathrm{Se}_{0.1}$ and $\mathrm{GaV}_{4} \mathrm{~S}_{7.8} \mathrm{Se}_{0.2}$ [Fig. 2(g)] which show that

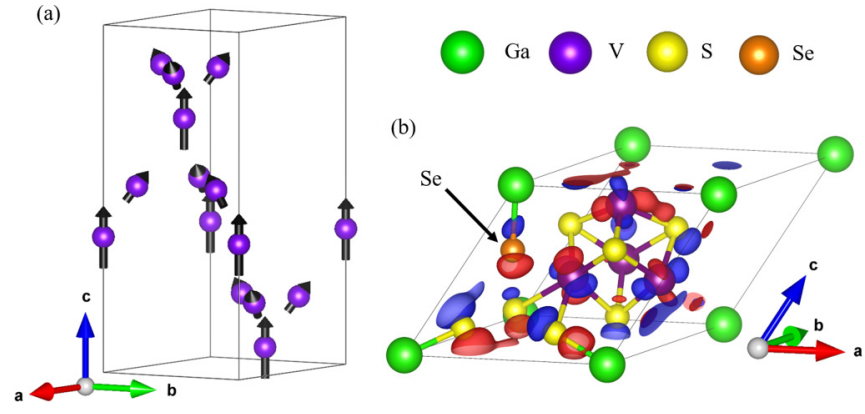

FIG. 3. (a) FM* ground state for $\mathrm{GaV}_{4} \mathrm{~S}_{8}$ (V atoms are shown.) (b) Difference in spin density between $y=0$ and $y=1$ for $\mathrm{Se}$ substitution on the $\mathrm{S}_{3}$ site from DFT.

the signature $\mathrm{FM}^{*}$ peak around $25 \mathrm{mT}$ is further suppressed upon increased substitution with spectral weight shifting to the broad high-field peak not present in $\mathrm{GaV}_{4} \mathrm{~S}_{8}$. As the $B_{3}$ component becomes more pronounced with increased $\mathrm{Se}$ substitution this suggests the increase in spin density is not caused by muon implantation during the measurements.

The Se-rich end of the series shows more conventional magnetic behavior with $\mu \mathrm{SR}$ measurements on the $y=8$ material suggesting $T_{\mathrm{c}}=17.5(5) \mathrm{K}$ with FT spectra consistent with simulations of a cycloidal spin structure [32]. Measurements of $\mathrm{GaV}_{4} \mathrm{~S}_{0.1} \mathrm{Se}_{7.9}$ show similar average fields but also feature an additional relaxing component and larger relaxation rates, indicating a broadening of the local magneticfield distribution.

To further understand the effect of substitution, we performed DFT calculations (see Ref. [32]) comparing the pristine materials to substitution of $y=1$ or $y=7$ (which are not measured here) by replacing the atoms on one $\mathrm{S}$ or $\mathrm{Se}$ site with the relevant substituent. This allows us to simulate the effect of low levels of substitution. We compare the spin density in electron bands that dominate the contribution to the magnetism (i.e., those occupied in only one spin channel) with the difference between $y=0$ and $y=1$ for the $S_{3}$ site shown in Fig. 3(b). Regardless of the site chosen for substitution, more dramatic changes in spin density are seen at the $S$ end of the series than at the Se end. Whereas most of the change in spin density retains the expected $d$-orbital character, some site substitutions result in an increase in spin density within the $\mathrm{V}$ tetrahedral cluster. Since there are muon-stopping sites around the cluster, this provides an explanation for the observed large magnetic-field $B_{3}$. The change in spin density may also lead to altered exchange pathways, which, upon increased substitution, could lead to the glasslike magnetic ground state seen for $y=2$ and $y=4$ [21] where multiple different exchange pathways (depending on the local substitution level) cannot be simultaneously satisfied.

We next explore the SkL state that appears in an applied field through transverse-field (TF) and longitudinalfield (LF) $\mu \mathrm{SR}$ [32]. Samples were cooled in zero applied magnetic field, and the measurements performed in the field (as indicated in Fig. 1) on warming. TF measurements are mainly sensitive to static disorder along with the component of dynamic fluctuations of the local field parallel to the applied field, whereas LF measurements are sensitive 

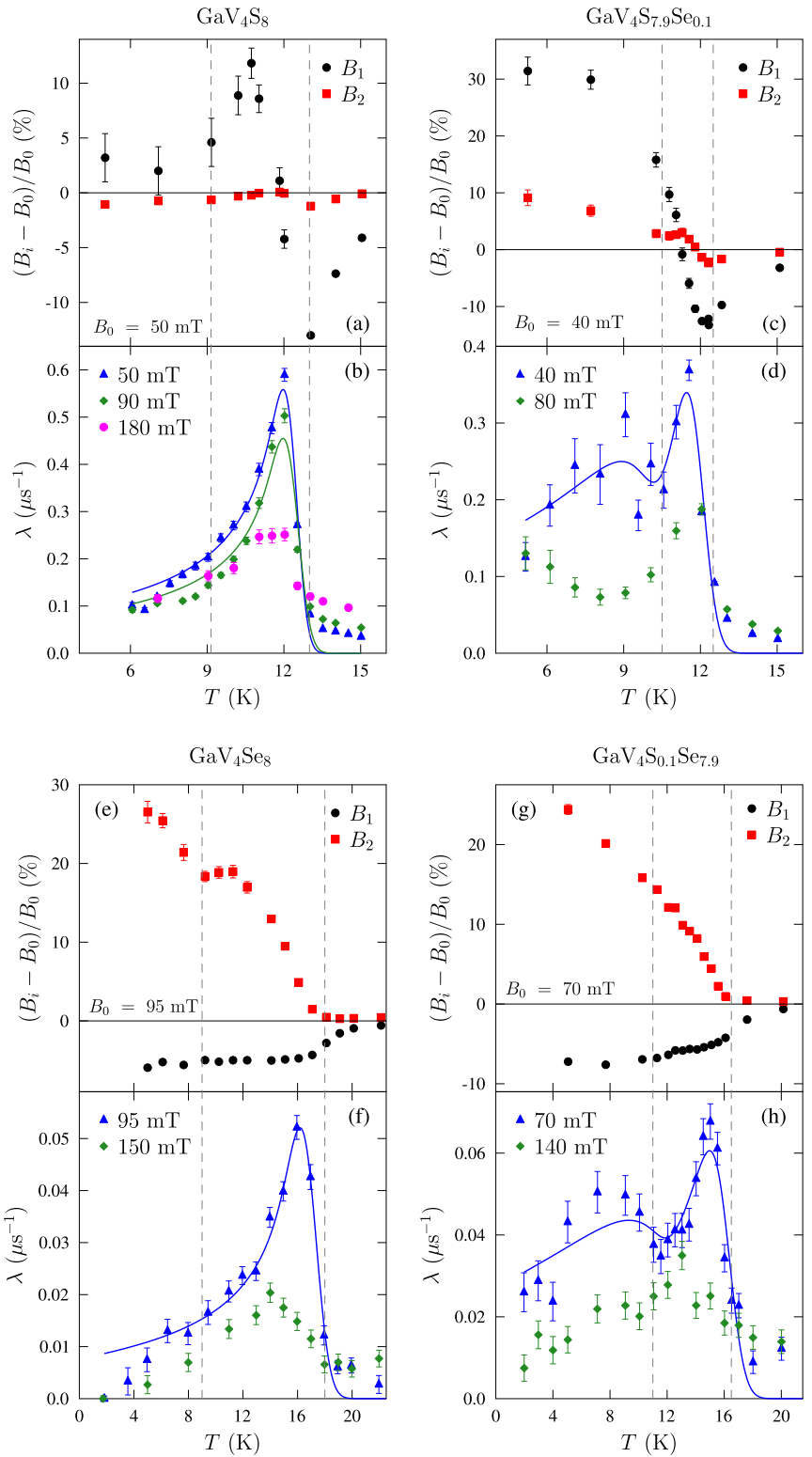

FIG. 4. Parameters from TF $\mu$ SR measurements on (a) $y=0$, (c) $y=0.1$, (e) $y=8$, and (g) $y=7.9$; and LF measurements for (b) $y=0$, (d) $y=0.1$, (f) $y=8$, and (h) $y=7.9$. Dashed lines are suggested boundaries for the SkL. Fits in (b), (d), (f), and (h) are detailed in the text.

to dynamics in those local fields perpendicular to the applied field. The SkL orientation, determined predominantly by the crystalline anisotropy, will be randomized in a polycrystalline sample, such as ours, even under application of an external magnetic field, and, hence, the two techniques are expected to be sensitive to the same dynamic field correlations. For the TF measurements, the data are described by $P_{x}(t)=\sum_{i=1}^{2} a_{i} e^{-\Lambda_{i} t} \cos \left(\gamma_{\mu} B_{i} t+\phi_{i}\right)+a_{\mathrm{b}}$ with results shown in Fig. 4 and Ref. [32].

In $\mathrm{GaV}_{4} \mathrm{~S}_{8}$, a peak in $B_{1}$ is seen at $9 \lesssim T \lesssim 12 \mathrm{~K}$ [Fig. 4(a)], coinciding with the presence of the SkL. This is consistent with $\mu \mathrm{SR}$ of the SkL in materials, such as $\mathrm{Cu}_{2} \mathrm{OSeO}_{3}$ [37] where an additional high-field shoulder is a signature of the SkL. LF data for $\mathrm{GaV}_{4} \mathrm{~S}_{8}$ are well parametrized by $P_{z}(t) \propto a e^{-\lambda t}+a_{\mathrm{b}}$ over the entire temperature range, consistent with dynamic relaxation. Measurements performed in applied fields of 50 and $90 \mathrm{mT}$ cut through the SkL state in, at least, some crystal orientations [17], whereas measurements made at $180 \mathrm{mT}$ do not. These data [Fig. 4(b)] show that the effect of the dynamics in the SkL state is a significant enhancement in relaxation rate $\lambda$ below $T_{\mathrm{c}}$ leading to a large broadened peak centered at temperatures within the SkL state. This is also consistent with LF measurements of the $\mathrm{SkL}$ in $\mathrm{Cu}_{2} \mathrm{OSeO}_{3}$ [38]. We attribute the enhanced $\lambda$ to skyrmion excitation modes with frequency $v$ (such as the low-frequency rotational and breathing modes in the SkL plane [39]) that soften (decreasing in frequency) as $T$ increases towards $T_{\mathrm{c}}[40,41]$. As these modes cross through the frequency window where $\mu \mathrm{SR}$ is sensitive (around the Larmor resonance frequency $\left.\omega_{0}=\gamma_{\mu} B_{\text {ext }}\right)$ the relaxation rate increases. In the fast fluctuation regime $\lambda=2 \Delta^{2} v /\left(\omega_{0}^{2}+v^{2}\right)$ where $\Delta$ is the width of the local field at the muon site. Applying power-law behavior typical for a threedimensional Heisenberg magnet $v=v_{0}\left(1-T / T_{\mathrm{c}}\right)^{1.43}$ and $\Delta=\Delta_{0}\left[1-\left(T / T_{\mathrm{c}}\right)^{3 / 2}\right]^{0.365}[32,42-45]$ produces good fits of $\lambda$ as seen in Fig. 4(b). We find the zero-temperature skyrmion excitation mode frequency is approximately $10 \mathrm{GHz}$, consistent with the $3-17 \mathrm{GHz}$ range observed in Bloch skyrmion materials [39] and similar to the frequencies measured in single crystals of $\mathrm{GaV}_{4} \mathrm{~S}_{8}$ [46].

The behavior of $\mathrm{GaV}_{4} \mathrm{Se}_{8}$ is similar to that of $\mathrm{GaV}_{4} \mathrm{~S}_{8}$ with a peak observed in the TF-field component $B_{2}$ [Fig. 4(e)]. LF $\mu$ SR measurements on $\mathrm{GaV}_{4} \mathrm{Se}_{8}$ in an applied field of $95 \mathrm{mT}$, [Fig. 4(f)] also shows a significantly enhanced relaxation rate in the SkL state (and peak below $T_{\mathrm{c}}$ ) when compared to the temperature scan with an applied field of $150 \mathrm{mT}$ where the SkL state is not stabilized. This confirms the suggestion in Ref. [21] that the SkL in polycrystalline samples is confined to a smaller region of the phase diagram than in singlecrystal samples. A frequency of around $16 \mathrm{GHz}$ for the zerotemperature excitation mode is found, again consistent with other skyrmion materials.

Finally, we turn to the influence of low levels of substitution on the SkL state. TF measurements for $\mathrm{GaV}_{4} \mathrm{~S}_{7.9} \mathrm{Se}_{0.1}$ were performed in an applied field of $40 \mathrm{mT}$ where an enhanced AC susceptibility response is consistent with a SkL state existing. Unlike in the $\mathrm{GaV}_{4} \mathrm{~S}_{8}$ case, no unambiguous signature of the SkL state is observed in the internal magnetic field. In fact, the field $B_{1}$ is significantly larger for $y=0.1$ compared to $y=0$, consistent with the large internal field observed in our ZF $\mu$ SR measurements. This implies that any peak in an internal field arising in the presence of the SkL state will be masked by these large fields. The variation in $B_{2}$ is similar between the samples, suggesting the underlying behavior is similar. However, LF $\mu \mathrm{SR}$ measurements on the $y=0.1$ material [Fig. 4(d)] show a significantly enhanced peak in $\lambda$ at $40 \mathrm{mT}$ compared to measurements at $80 \mathrm{mT}$, strongly suggestive of the characteristic dynamics of the SkL state. This is accompanied by a region of enhanced $\lambda$ at lower temperature, observed only at fields where the SkL response is found.

At the Se-rich end of the series, fits of TF $\mu$ SR measurements for $y=7.9$ in an applied field of $70 \mathrm{mT}$ where an 
enhanced AC susceptibility response consistent with a SkL is seen just below $T_{\mathrm{c}}$ are shown in Fig. $4(\mathrm{~g})$. Although the overall trends in behavior are similar to those for $y=8$, there is again no resolvable peak in internal field in the SkL region. However, LF $\mu$ SR measurements [Fig. 4(h)] show that there is a clear enhanced response in $\lambda$ at $70 \mathrm{mT}$ compared to $140 \mathrm{mT}$, consistent with the realization of the SkL state at $70 \mathrm{mT}$. In addition, we again observe a separate enhancement in the low-temperature relaxation rate, similar to the behavior observed in $y=0.1$ with a broad peak in the relaxation rate centered around $T=8 \mathrm{~K}$.

The second lower-temperature peak in $\lambda$ that appears upon substitution only at fields at which the SkL is stabilized suggests that there are dynamics associated with the SkL extending down to lower temperatures. We propose that these dynamics occur due to skyrmion precursors (as seen in $\mathrm{Cu}_{2} \mathrm{OSeO}_{3}$ [47]) that are stabilized by the subsituents at both ends of the series. Note that if the dynamics were associated with the cycloidal phase, one would expect a peak in $\lambda$ at all applied fields that stabilize the cycloidal phase. The stabilization of the skyrmion precursors either due to increased formation caused by pinning or to longer lifetimes of metastable states leads to dynamics detectable with $\mu$ SR that are not dominant in the pristine materials. These dynamics likely arise from a reduced frequency $\mathrm{SkL}$ mode due to the $\approx 300$ substituents per skyrmion present in the substituted systems, making the skyrmions less rigid, and, hence, lowering their characteristic frequencies. Fitting this two-mode model for $\lambda$ [Figs. 4(d) and 4(h)] reveals that the low- $T$ mode frequency is approximately $10-20 \%$ of the high- $T$ frequency without a significant change in the width of the local field.
In conclusion, our results show that magnetic order is preserved for low levels of substitution in $\mathrm{GaV}_{4} \mathrm{~S}_{8-y} \mathrm{Se}_{y}$ at both ends of the series in contrast to higher levels where spin glasslike behavior is observed [21,22]. On the Se-rich end of the series, the presence of $\mathrm{S}$ simply leads to an increase in the width of the local magnetic-field distribution, which is enough to prevent observation of the effect of the SkL in our TF measurements. On the S-rich end of the series where the ZF-ordered state is formed from competing $\mathrm{FM}^{*}$ and $\mathrm{C}$ orders, substitution has a more dramatic effect, creating regions in the sample which have increased spin density, leading to the observation of high magnetic fields with $\mu$ SR. At both ends of the series, LF $\mu$ SR provides evidence of enhanced dynamics typical of those observed in Néel SkL states. We, therefore, conclude that a dynamically fluctuating $\mathrm{SkL}$ is realized in these materials with low levels of chemical substitution with skyrmion precursors at temperatures below the equilibrium skyrmion lattice. We have shown further that the zero-temperature frequency of the Néel SkL excitation modes appear to be similar to those for a Bloch SkL and suggest that skyrmion precursors may be ubiquitous over a wide range of temperatures in SkL materials.

Part of this work was carried our at the STFC ISIS Facility, UK, and part of this work was carried out at the Swiss Muon Source $(\mathrm{S} \mu \mathrm{S})$, Paul Scherrer Institut, Switzerland, and we are grateful for the provision of beamtime. This project was funded by EPSRC (UK) (Grant Nos: EP/N032128/1 and EP/N024028/1). M.G. would like to acknowledge the Slovenian Research Agency under Project No. Z1-1852. Research data from this paper will be made available via Durham Collections at doi: 10.15128/r1b2773v71x.
[1] P. A. Lee, N. Nagaosa, and X.-G. Wen, Doping a Mott insulator: Physics of high-temperature superconductivity, Rev. Mod. Phys. 78, 17 (2006).

[2] N. P. Butch and M. B. Maple, The suppression of hidden order and the onset of ferromagnetism in $\mathrm{URu}_{2} \mathrm{Si}_{2}$ via Re substitution, J. Phys.: Condens. Matter 22, 164204 (2010).

[3] M. N. Wilson, T. J. Williams, Y.-P. Cai, A. M. Hallas, T. Medina, T. J. Munsie, S. C. Cheung, B. A. Frandsen, L. Liu et al., Antiferromagnetism and hidden order in isoelectronic doping of $\mathrm{URu}_{2} \mathrm{Si}_{2}$, Phys. Rev. B 93, 064402 (2016).

[4] M. N. Wilson, Muon spin rotation studies of $\mathrm{URu}_{2} \mathrm{Si}_{2}$ and dichalcogenide superconductors, Ph.D. thesis, McMaster University, Canada (2018).

[5] J. Kondo, Resistance minimum in dilute magnetic alloys, Prog. Theor. Phys. 32, 37 (1964).

[6] A. C. Hewson, The Kondo Problem to Heavy Fermions (Cambridge University Press, Cambridge, 1997).

[7] M. Gomilšek, R. Žitko, M. Klanjšek, M. Pregelj, C. Baines, Y. Li, Q. Zhang, and A. Zorko, Kondo screening in a chargeinsulating spinon metal, Nat. Phys. 15, 754 (2019).

[8] K. Everschor-Sitte, J. Masell, R. M. Reeve, and M. Kläui, Perspective: Magnetic skyrmions - overview of recent progress in an active research field, J. Appl. Phys. 124, 240901 (2018).

[9] T. Lancaster, Skyrmions in magnetic materials, Contemp. Phys. 60, 246 (2019).
[10] M. T. Birch, R. Takagi, S. Seki, M. N. Wilson, F. Kagawa, A. Štefančič, G. Balakrishnan, R. Fan, P. Steadman, C. J. Ottley, M. Crisanti, R. Cubitt, T. Lancaster, Y. Tokura, and P. D. Hatton, Increased lifetime of metastable skyrmions by controlled doping, Phys. Rev. B 100, 014425 (2019).

[11] M. N. Wilson, M. Crisanti, C. Barker, A. Štefančič, J. S. White, M. T. Birch, G. Balakrishnan, R. Cubitt, and P. D. Hatton, Measuring the formation energy barrier of skyrmions in zincsubstituted $\mathrm{Cu}_{2} \mathrm{OSeO}_{3}$, Phys. Rev. B 99, 174421 (2019).

[12] S. Mühlbauer, B. Binz, F. Jonietz, C. Pfleiderer, A. Rosch, A. Neubauer, R. Georgii, and P. Böni, Skyrmion lattice in a chiral magnet, Science 323, 915 (2009).

[13] W. Münzer, A. Neubauer, T. Adams, S. Mühlbauer, C. Franz, F. Jonietz, R. Georgii, P. Böni, B. Pedersen, M. Schmidt et al., Skyrmion lattice in the doped semiconductor $\mathrm{Fe}_{1-x} \mathrm{Co}_{x} \mathrm{Si}$, Phys. Rev. B 81, 041203(R) (2010).

[14] X. Yu, N. Kanazawa, Y. Onose, K. Kimoto, W. Z. Zhang, S. Ishiwata, Y. Matsui, and Y. Tokura, Near room-temperature formation of a skyrmion crystal in thin-films of the helimagnet FeGe, Nature Mater. 10, 106 (2011).

[15] S. Seki, X. Z. Yu, S. Ishiwata, and Y. Tokura, Observation of skyrmions in a multiferroic material, Science 336, 198 (2012).

[16] T. Kurumaji, T. Nakajima, M. Hirschberger, A. Kikkawa, Y. Yamasaki, H. Sagayama, H. Nakao, Y. Taguchi, T.-h. Arima, and Y. Tokura, Skyrmion lattice with a giant topological Hall 
effect in a frustrated triangular-lattice magnet, Science $\mathbf{3 6 5}, 914$ (2019).

[17] I. Kézsmárki, S. Bordács, P. Milde, E. Neuber, L. M. Eng, J. S. White, H. M. Rønnow, C. D. Dewhurst, M. Mochizuki, K. Yanai et al., Néel-type skyrmion lattice with confined orientation in the polar magnetic semiconductor $\mathrm{GaV}_{4} \mathrm{~S}_{8}$, Nature Mater. 14, 1116 (2015).

[18] Y. Fujima, N. Abe, Y. Tokunaga, and T. Arima, Thermodynamically stable skyrmion lattice at low temperatures in a bulk crystal of lacunar spinel $\mathrm{GaV}_{4} \mathrm{Se}_{8}$, Phys. Rev. B 95, 180410(R) (2017).

[19] T. Kurumaji, T. Nakajima, V. Ukleev, A. Feoktystov, T. H. Arima, K. Kakurai, and Y. Tokura, Néel-Type Skyrmion Lattice in the Tetragonal Polar Magnet $\operatorname{VOSe}_{2} \mathrm{O}_{5}$, Phys. Rev. Lett. 119, 237201 (2017).

[20] A. K. Srivastava, P. Devi, A. K. Sharma, T. Ma, H. Deniz, H. L. Meyerheim, C. Felser, and S. S. P. Parkin, Observation of robust Néel skyrmions in metallic PtMnGa, Adv. Mater. 32, 1904327 (2019).

[21] K. J. A. Franke, B. M. Huddart, T. J. Hicken, F. Xiao, S. J. Blundell, F. L. Pratt, M. Crisanti, J. A. T. Barker, S. J. Clark, A. Štefančič et al., Magnetic phases of skyrmion-hosting $\mathrm{GaV}_{4} \mathrm{~S}_{8-y} \mathrm{Se}_{y}(y=0,2,4,8)$ probed with muon spectroscopy, Phys. Rev. B 98, 054428 (2018).

[22] A. Štefančič, S. J. R. Holt, M. R. Lees, C. Ritter, M. J. Gutmann, T. Lancaster, and G. Balakrishnan, Establishing magneto-structural relationships in the solid solutions of the skyrmion hosting family of materials: $\mathrm{GaV}_{4} \mathrm{~S}_{8-y} \mathrm{Se}_{y}$, Sci. Rep. 10, 9813 (2020).

[23] S. J. Blundell, Spin-polarized muons in condensed matter physics, Contemp. Phys. 40, 175 (1999).

[24] C. V. Topping and S. J. Blundell, AC susceptibility as a probe of low-frequency magnetic dynamics, J. Phys.: Condens. Matter 31, 013001 (2018).

[25] S. J. Clark, M. D. Segall, C. J. Pickard, P. J. Hasnip, M. J. Probert, K. Refson, and M. Payne, First principles methods using CASTEP, Z. Kristallogr. Cryst. Mater. 220, 567 (2005).

[26] J. P. Perdew, K. Burke, and M. Ernzerhof, Generalized Gradient Approximation Made Simple, Phys. Rev. Lett. 77, 3865 (1996).

[27] H. J. Monkhorst and J. D. Pack, Special points for Brillouinzone integrations, Phys. Rev. B 13, 5188 (1976).

[28] H. Müller, W. Kockelmann, and D. Johrendt, The magnetic structure and electronic ground states of mott insulators $\mathrm{GaV}_{4} \mathrm{~S}_{8}$ and $\mathrm{GaV}_{4} \mathrm{Se}_{8}$, Chem. Mater. 18, 2174 (2006).

[29] J. T. Zhang, J. L. Wang, X. Q. Yang, W. S. Xia, X. M. Lu, and J. S. Zhu, Magnetic properties and spin-driven ferroelectricity in multiferroic skyrmion host $\mathrm{GaV}_{4} \mathrm{~S}_{8}$, Phys. Rev. B 95, 085136 (2017).

[30] F. L. Pratt, WiMDA: A muon data analysis program for the Windows PC, Physica B 289, 710 (2000).

[31] P. Bonfà, I. J. Onuorah, and R. De Renzi, Introduction and a quick look at MUESR, the Magnetic structure and mUon Embedding Site Refinement suite, Proceedings of the 14th International Conference on Muon Spin Rotation, Relaxation and Resonance ( SSR2017), Sapporo, Japan, 2018, (Japan Physical Society, Tokyo, Japan, 2018), p. 011052.

[32] See Supplemental Material at http://link.aps.org/supplemental/ 10.1103/PhysRevResearch.2.032001 for detailed methodology, derivations, and raw data with other fitted parameters.
[33] E. Ruff, S. Widmann, P. Lunkenheimer, V. Tsurkan, S. Bordács, I. Kézsmárki, and A. Loidl, Multiferroicity and skyrmions carrying electric polarization in $\mathrm{GaV}_{4} \mathrm{~S}_{8}$, Sci. Adv. 1, e1500916 (2015).

[34] J. S. White, A. Butykai, R. Cubitt, D. Honecker, C. D. Dewhurst, L. F. Kiss, V. Tsurkan, and S. Bordács, Direct evidence for cycloidal modulations in the thermal-fluctuationstabilized spin spiral and skyrmion states of $\mathrm{GaV}_{4} \mathrm{~S}_{8}$, Phys. Rev. B 97, 020401(R) (2018).

[35] E. M. Clements, R. Das, G. Pokharel, M. H. Phan, A. D. Christianson, D. Mandrus, J. C. Prestigiacomo, M. S. Osofsky, and H. Srikanth, Robust cycloid crossover driven by anisotropy in the skyrmion host $\mathrm{GaV}_{4} \mathrm{~S}_{8}$, Phys. Rev. B 101, 094425 (2020).

[36] Y. A. Izyumov, Modulated, or long-periodic, magnetic structures of crystals, Sov. Phys. Usp. 27, 845 (1984).

[37] T. Lancaster, R. C. Williams, I. O. Thomas, F. Xiao, F. L. Pratt, S. J. Blundell, J. C. Loudon, T. Hesjedal, S. J. Clark, P. D. Hatton et al., Transverse field muon-spin rotation signature of the skyrmion-lattice phase in $\mathrm{Cu}_{2} \mathrm{OSeO}_{3}$, Phys. Rev. B 91, 224408 (2015).

[38] A. Štefančič, S. H. Moody, T. J. Hicken, M. T. Birch, G. Balakrishnan, S. A. Barnett, M. Crisanti, J. S. O. Evans, S. J. R. Holt, K. J. A. Franke et al., Origin of skyrmion lattice phase splitting in zn-substituted $\mathrm{Cu}_{2} \mathrm{OSeO}_{3}$, Phys. Rev. Mater. 2, 111402 (2018).

[39] M. Garst, J. Waizner, and D. Grundler, Collective spin excitations of helices and magnetic skyrmions: Review and perspectives of magnonics in non-centrosymmetric magnets, J. Phys. D: Appl. Phys. 50, 293002 (2017).

[40] S. Seki, Y. Okamura, K. Shibata, R. Takagi, N. D. Khanh, F. Kagawa, T. Arima, and Y. Tokura, Stabilization of magnetic skyrmions by uniaxial tensile strain, Phys. Rev. B 96, 220404(R) (2017).

[41] R. Tomasello, A. Giordano, S. Chiappini, R. Zivieri, G. Siracusano, V. Puliafito, I. Medlej, A. La Corte, B. Azzerboni, M. Carpentieri et al., Micromagnetic understanding of the skyrmion Hall angle current dependence in perpendicularly magnetized ferromagnets, Phys. Rev. B 98, 224418 (2018).

[42] S. J. Blundell, Magnetism in Condensed Matter (Oxford University Press, Oxford, 2003).

[43] R. Pełka, D. Pinkowicz, B. Sieklucka, and M. Fitta, Molecular realizations of 3D Heisenberg magnet: Critical scaling, J. Alloys Compd. 765, 520 (2018).

[44] M. Troyer, M. Imada, and K. Ueda, Critical exponents of the quantum phase transition in a planar antiferromagnet, J. Phys. Soc. Jpn. 66, 2957 (1997).

[45] E. Pospelov, V. Prudnikov, P. Prudnikov, and A. Lyakh, Nonequilibrium critical behavior of the 3D classical Heisenberg model, J. Phys.: Conf. Ser. 1163, 012020 (2019).

[46] D. Ehlers, I. Stasinopoulos, V. Tsurkan, H. A. Krugvon Nidda, T. Fehér, A. Leonov, I. Kézsmárki, D. Grundler, and A. Loidl, Skyrmion dynamics under uniaxial anisotropy, Phys. Rev. B 94, 014406 (2016).

[47] L. J. Bannenberg, H. Wilhelm, R. Cubitt, A. Labh, M. P. Schmidt, E. Lelièvre-Berna, C. Pappas, M. Mostovoy, and A. O. Leonov, Multiple low-temperature skyrmionic states in a bulk chiral magnet, npj Quantum Mater. 4, 11 (2019). 